\title{
The 20S proteasome core, active within apoptotic exosome-like vesicles, induces autoantibody production and accelerates rejection
}

Melanie Dieudé $\dagger^{1,6}$, Christina Bell $\dagger^{2,6} \ddagger$, Julie Turgeon ${ }^{1,6}$, Deborah Beillevaire ${ }^{1,6}$, Luc Pomerleau ${ }^{1,6}$, Bing Yang ${ }^{1,6}$, Katia Hamelin ${ }^{1,6}$, Shijie Qi ${ }^{1,6}$, Nicolas Pallet ${ }^{1} \ddagger$, Chanel Béland ${ }^{1}$, Wahiba Dhahri ${ }^{1}$, Jean-Francoi. Cailhier ${ }^{1,6}$, Matthieu Rousseau ${ }^{3,6}$, Anne-Claire Duchez ${ }^{3,6}$, Tania Lévesque ${ }^{3,6}$, Arthur Lau, ${ }^{5,6}$, Christiane Rondeau $^{4}$, Diane Gingras ${ }^{4}$, Danie Muruve ${ }^{5,6}$, Alain Rivard ${ }^{1}$, Heloise Cardinal ${ }^{1,6}$, Claude Perreault ${ }^{2,6}$, Michel Desjardins ${ }^{4}$, Eric Boilard ${ }^{3,6}$, Pierre Thibault*2,6 and Marie-Josée Hébert*1,6

${ }^{1}$ Research Centre, Centre hospitalier de l'Université de Montréal (CRCHUM).

${ }^{2}$ Institute for research in immunology and cancer (IRIC).

${ }^{3}$ Centre de Recherche du CHU de Québec

${ }^{4}$ Université de Montréal

5 University of Calgary

${ }^{6}$ Canadian National Transplantation Research Program

* Both authors contributed equally to the work

$\dagger$ Both authors contributed equally to the work

$\ddagger$ Christina Bell's current affiliation: Department of Cell Biology, Harvard Medical School, Boston, MA

02115, USA, Nicolat Pallet current affiliation: INSERM U1147, 75006 Paris, France

${ }^{*}$ Dr. Marie-Josée Hébert, CRCHUM, Centre de Recherche du CHUM, Tour Viger - R12.414, 900 rue St-Denis, H2X 0A9, Montréal (QC)

Tel:(514) 890-8000 Extension 25393, Fax: (514) 412-7661.

E-mail: marie-josee.hebert.chum@ssss.gouv.qc.ca

${ }^{*}$ Dr. Pierre Thibault, IRIC, Universite de Montreal,

P.O. Box 6128, Station Centre-ville, H3C3J7, Montréal (QC)

Tel (514) 343-6910, Fax: (514) 343-6843.

E-mail: pierre.thibault@umontreal.ca

$\dagger$ Both authors contributed equally to the work

One sentence summary: Exosome-like vesicles containing an active $20 \mathrm{~S}$ proteasome core contribute to autoimmunity and vascular allograft inflammation. 
Autoantibodies to components of apoptotic cells, such as anti-perlecan antibodies, contribute to rejection in organ transplant recipients. However, mechanisms of immunization to apoptotic components remain largely uncharacterized. Here, we used large-scale proteomics, with validation by electron microscopy and biochemical methods, to compare the protein profiles of apoptotic bodies and apoptotic exosomelike vesicles, smaller extracellular vesicles released by endothelial cells downstream of caspase-3 activation. We identified apoptotic exosome-like vesicles as a central trigger for production of anti-perlecan antibodies and acceleration of rejection. Unlike apoptotic bodies, apoptotic exosome-like vesicles triggered the production of anti-perlecan antibodies in naïve mice and enhance anti-perlecan antibody production and allograft inflammation in mice transplanted with a $\mathrm{MHC}$-incompatible aortic graft. The $20 \mathrm{~S}$ proteasome core was active within apoptotic exosome-like vesicles and controlled their immunogenic activity. Finally, we showed that proteasome activity in circulating exosome-like vesicles increased following vascular injury in mice. These findings open new avenues for predicting and controlling maladaptive humoral responses to apoptotic cell components that enhance risk of rejection post-transplantation. 


\section{Introduction}

Antibodies targeting the vasculature of solid allografts are major risk factors of poor outcomes in solid organ transplantation (1-7). Although donor specific anti-human leucocyte antigen antibodies (DSA) play a major role in allograft vascular injury, autoantibodies are increasingly recognized as important contributors to allograft vascular rejection and decreased long-term survival (8-19). Autoantibodies to vimentin, angiotensin II type 1 receptor, collagen V, tubulin, fibronectin and perlecan/LG3 have been associated with increased rejection rates and reduced survival of heart, kidney and lung allografts $(8,17,20-28)$. The classic view in the field was that autoantibody titers increase during acute rejection episodes secondary to the release of danger associated molecular patterns (DAMPs). However, recent studies have reported the presence of autoantibodies prior to transplantation in de novo transplant patients, excluding rejection as a sine qua non factor for production of auto-antibodies (8). AntiLG3 and anti-angiotensin receptors have been detected in untransfused ${ }^{*}$ untransplanted males and untransfused ${ }^{*} u n t r a n s p l a n t e d$ females who have never been pregnant $(8,29)$, also suggesting that allo-sensitizing events are not required for their production $(8,17$, 20-25).

Abnormal reactivity to apoptotic cells plays a role in fuelling the production of at least some of these autoantibodies. Two recent studies have identified circulating levels of IgG reactive with apoptotic cells as a marker of antibody-mediated rejection (ABMR) $(30,31)$ in renal transplant patients. Remarkably, the detection, pre-transplantation, of antibodies reactive with apoptotic cells predicted increased risk of ABMR and reduced 
long-term allograft survival. Our group also described an association between pretransplantation titers of $\lg G$ autoantibodies against the C-terminal perlecan fragment LG3 and the risk of acute vascular rejection in renal transplant patients (8). Apoptosis of endothelial cells is a major pathway regulating perlecan proteolysis and production of LG3 $(32,33)$. The association between autoantibodies and apoptosis is intriguing, given the classical notion that apoptosis represents a non-immunogenic if not tolerogenic type of regulated cell death $(34,35)$. Apoptotic cells are known to release apoptotic bodies, which, when engulfed by professional phagocytes, trigger the secretion of antiinflammatory cytokines (e.g. TGF- $\beta, \mathrm{IL}-10)(34,36)$ and increase numbers of regulatory T cells. Autoantibodies to components of apoptotic cells or apoptotic bodies have been identified in lupus patients (37), due to abnormalities in clearance of apoptotic cells that prevent phagocytosis-induced immune tolerance and lead to secondary necrosis (34). However, no propensity towards lupus or other autoimmune diseases was found in patients with autoantibodies against apoptotic cells or perlecan/LG3 $(8,30)$.

Recent work by our group and others has highlighted a major role for apoptosis and caspase-3 activation in controlling the release of extracellular vesicles (EVs) that appear to differ from classical apoptotic bodies (38-40). Apoptotic bodies, classically ranging in size from 1-5 um and characterized by the presence of histone components, are released by apoptotic cells through caspase-dependent pathways (41). Caspase activation can also lead to the release of smaller EVs, referred to as apoptotic exosomelike vesicles and ranging in size from 30 to $100 \mathrm{~nm}$. The immune modulating functions of apoptotic exosome-like vesicles are still largely uncharacterized. 
Here we aim to evaluate the immunogenic potential of the various extracellular structures released by apoptotic cells (ECs) downstream of caspase-3 activation in the early phases of apoptosis. We use large scale proteomics to profile the protein signatures of apoptotic bodies and apoptotic exosome-like vesicles. In addition, we compare the capacity of apoptotic exosome-like vesicles and apoptotic bodies to trigger the production of autoantibodies in transplanted and non-transplanted mice and shape the severity of rejection. 


\section{Results}

Apoptotic endothelial cells release different types of EVs downstream of caspase-3 activation

To characterize the various types of EVs released by apoptotic ECs, human umbilical vein endothelial cells (HUVECs) or primary murine aortic endothelial cells (mECs) were induced to undergo apoptosis through exposure to serum-free medium in vitro. In keeping with the translational scope of our work, we chose serum starvation for stimulation of apoptosis as exposure to serum free preservation solution before transplantation is an integral component of all types of organ transplantations. In both cell types, serum deprivation significantly increased caspase-3 activation and the percentage of cells with chromatin condensation in absence of cell membrane permeabilization (Supplementary figure $1 \mathrm{a}$ and $\mathrm{b}$ ). These results are consistent with previous work by our group demonstrating that this system induces apoptosis in the absence of primary or secondary necrosis $(39,42)$. Preventing activation of caspases and, more specifically, caspase-3 through biochemical inhibition or the use of mECs from caspase-3 - ${ }^{-}$mice significantly reduced the development of apoptosis (Supplementary figures $1 \mathrm{a}$ and $1 \mathrm{~b}$ ). Medium conditioned by serum-starved HUVEC or mEC was harvested at time points associated with caspase-3 activation and evaluated for the presence of EVs (Figures 1a and 1b). Using a high sensitivity flow cytometer equipped with a forward scatter coupled to a photomultiplier tube designed to resolve smaller particles (Supplementary Figure 2) $(43,44)$, we confirmed that vesicles of various sizes are released by endothelial cells during apoptosis (Figures 1a and 1b). The vast majority of detected particles (95-99\%) were sensitive to dissolution by the 
detergent Triton $\mathrm{X}-100$, confirming that these structures are bound by a lipid membrane (Figures $1 \mathrm{a}, 1 \mathrm{~b}$ and supplementary figure 3 ).

As previously published, serum starved ECs engage a mixed autophagic and apoptotic response without any necrosis $(39,42)$. Preventing autophagy through biochemical inhibition with bafilomycin A1 did not modulate the release of extracellular vesicles (Supplementary figure 4). However, pan-caspase inhibition, specific caspase-3 inhibition or the use of endothelial cells from caspase $-3^{-}$mice led to a significantly reduced production of apoptotic exosome-like vesicles and to a lesser extend apoptotic bodies (Figures 1a and 1b) highlighting the importance of caspases in the secretion of EVs by serum starved ECs.

We then used sequential centrifugation of conditioned medium to isolate fractions enriched in apoptotic bodies or apoptotic exosome-like vesicles. The two fractions showed EVs with distinct ultrastructural characteristics (Figure 1c and supplementary figure 5 and 6). As reported previously (42), the majority of EVs isolated by centrifugation at $50,000 \mathrm{xg}$ were within the range of apoptotic bodies from 1 to $5 \mathrm{um}$, (containing intracytoplasmic components and various organelles, such as mitochondria) along with some smaller membrane vesicles within microvesicle size range (between $0.25 \mathrm{uM}$ to $1 \mathrm{uM}$ ). (Figure $1 \mathrm{c}$ and supplementary Figure 5). The fraction isolated by centrifugation at $200,000 \times \mathrm{xg}$ was enriched in smaller EVs showing a heterogeneous population of membrane-bound vesicles ranging in size from 30 to $100 \mathrm{~nm}$ (Figure 1c and supplementary Figure 6). Collectively, these results demonstrate that EVs of various sizes are released by apoptotic ECs through caspase-3-dependent pathways. 
Proteomics profiling of exosome-like vesicles and apoptotic bodies reveals distinct proteome signatures

To further characterize the differences between EVs released by apoptotic ECs, we performed a large-scale proteomics analysis and profiled the abundance of proteins in apoptotic bodies and apoptotic exosome-like vesicles. Apoptotic bodies and exosomelike vesicles were characterized by strikingly distinct protein markers (Figure 1d), confirming that exosome-like vesicles do not merely represent the debris of apoptotic bodies. Apoptotic bodies contain a higher number of proteins, with 546 identified proteins, while only 235 proteins were identified in exosome-like vesicles. The heatmap of protein abundance revealed that typical markers of apoptotic bodies such as histones (Histone H2A type 2-A. Histone $\mathrm{H} 4$ and Histone H2B type 2-E) were enriched in apoptotic bodies (Figure 1d).

We then performed gene ontology analysis to gain further insights into the processes by which proteins and pathways are actively modulated in the different EV subsets (Supplementary Figure 7). In line with our previous findings (42), apoptotic bodies were found to be enriched in ribosomal proteins and proteins from the cytosol, endoplasmic reticulum, nucleus and mitochondria. Exosome-like vesicles were enriched in extracellular matrix, basement membrane proteins and several lysosomal proteins (Supplementary Figure 7). More than $80 \%$ of the exosome-like vesicle proteome was listed in Exocarta and Vesiclepedia databases (Supplementary figure 8a and 8b) Also, $62 \%$ of the top 50 exosome protein markers according to Exocarta database were 
identified in exosome-like vesicles (Supplementary Table 1). Furthermore, western blot analyses comparing protein profiles of apoptotic bodies and apoptotic exosome-like vesicles showed enrichment in the exosome markers synthenin, fibronectin and TCTP (Supplementary figure 8c). Apoptotic bodies did not express characteristic exosome markers but showed increased expression of GM130 and tubulin. However, some classical exosome markers such as CD9, CD81, and TSG101 were not expressed in apoptotic exosome-like vesicles, as evidenced by proteomic analysis or western blotting, suggesting that these membrane vesicles, although closely related to exosomes, present some differences within protein signatures (Supplementary figure 8). Interestingly, the functions of proteins exclusive to exosome-like vesicles were associated with proteasomal degradation and ligase activities, while those of apoptotic bodies were enriched for RNA processing and vesicle targeting. More detailed analyses identified perlecan as one of the basement membrane proteins present in apoptotic exosome-like vesicles (Figure 1d and supplementary figure 7), with higher sequence coverage of the LG3 fragment of perlecan in exosome-like vesicles compared with apoptotic bodies (Figure 2a and supplementary Figure 9). Since LG3 and antibodies to LG3 have been associated with rejection in animal models and transplant patients $(8$, 45-47), we used electron microscopy with immunogold-labelling to further characterize the presence of LG3 in preparations of apoptotic exosome-like vesicles. Immunogold labelling identified LG3 in vesicular structures ranging in size from 30 to $100 \mathrm{~nm}$ (Figure 2b). To confirm that LG3 is released through caspase-dependent pathways, we assessed LG3 levels in preparations of exosome-like vesicles from caspase-inhibited or caspase $3^{-/}$ECs. Pan-caspase inhibition or the use of caspase- $3^{-}$ECs significantly 
reduced release of LG3 (Figures $2 \mathrm{c}$ and $2 \mathrm{~d}$ ). Collectively, these results demonstrate that apoptotic bodies and apoptotic exosome-like vesicles are characterized by distinct protein markers and that LG3 is a marker of apoptotic exosome-like vesicles.

Apoptotic exosome-like vesicles trigger anti-LG3 production.

We then evaluated whether apoptotic exosome-like vesicles can trigger the production of anti-LG3 autoantibodies and shape the severity of rejection. We first evaluated whether intravenous injection of apoptotic exosome-like vesicles fosters the production of anti-LG3 antibodies in wild-type naïve mice. Sequential centrifugation was used to purify apoptotic bodies and apoptotic vesicles from serum-free medium conditioned by $1 \times 10^{4}$ murine apoptotic ECs. Each fraction was then resuspended in half the initial volume of conditioned medium and injected to mice intravenously every other day for three weeks. Injection of apoptotic exosome-like vesicles but not apoptotic bodies significantly increased the production of anti-LG3 IgG antibodies in naïve mice (Figure 2e). Since total protein levels were found to be six times lower in preparations of apoptotic bodies compared with preparations of apoptotic exosome-like vesicles (2 $\mathrm{ug} / \mathrm{mL}$ and $0.3 \mathrm{ug} / \mathrm{mL}$, respectively), we also evaluated whether increasing the amount of injected apoptotic bodies would trigger the production of anti-LG3 antibodies. Injecting equal amounts of proteins from preparations of apoptotic bodies and preparations of apoptotic exosome-like vesicles failed to unmask immunogenic activity in apoptotic bodies (Supplementary figure 10). 
We then evaluated whether the injection of apoptotic exosome-like vesicles increases the production of other autoantibodies and markers of B cell activation. Anti-nuclear antibody (ANA) titers were increased in mice injected with exosome-like vesicles compared with apoptotic bodies or control mice (Supplementary figure 11a). Enhanced numbers of germinal centre B cells and follicular helper T cells were observed in mice injected with exosome-like vesicles (Supplementary figures 11b, 11c 11d and 11e). Collectively, these data suggest that apoptotic exosome-like vesicles, unlike apoptotic bodies, favor B cell responses associated, at least in part, with the production of autoantibodies.

Apoptotic exosome-like vesicles aggravate vascular rejection

We then compared the impact of apoptotic exosome-like vesicles and apoptotic bodies on the production of anti-LG3 antibodies and the severity of rejection in allografted mice. Apoptotic bodies and apoptotic exosome-like vesicles were purified from serum-free medium conditioned by $1 \times 10^{4}$ murine EC and injected intravenously every other day for three weeks post-transplantation into recipients of a fully MHC-mismatched aortic graft. Since we aimed to evaluate aggravation of rejection, allografts were studied at three weeks post-transplantation, a time point normally associated with minimal vascular remodelling and inflammation (Supplementary figure 12) $(46,47)$. The injection of transplanted mice with apoptotic exosome-like vesicles, but not apoptotic bodies, significantly increased the production of anti-LG3 IgG antibodies (Figure 3a) and heightened the severity of rejection (Figures $3 b, 3 c, 3 d, 3 e$ and3 f). 
Infiltration by CD3+ T cells and CD20+ B cells was significantly increased in mice injected with apoptotic exosome-like vesicles but not with apoptotic bodies (Figures 3b, 3d and 3e). C4d deposition, a hallmark of immune complex deposition, was also increased in mice injected with apoptotic exosome-like vesicles (Figures $3 b$ and $3 f$ ). Donor specific antibodies (DSAs) were detected in allografted mice but their levels were not affected by the injection of exosome-like vesicles or apoptotic bodies (Figure 3g). This suggests that apoptotic exosome-like vesicles accelerate rejection through immune complex-dependent but DSA-independent pathways. Anti-LG3 antibodies produced secondary to injection of apoptotic exosome-like vesicles are mainly of complementfixing isotypes (Supplementary figure 13). Collectively, these results demonstrate that apoptotic exosome-like vesicles, unlike apoptotic bodies, aggravate vascular allograft inflammation, C4d deposition and production of complement-fixing isotypes of anti-LG3 antibodies.

The proteasome core complex is enriched and active in apoptotic exosome-like vesicles

The observation that exosome-like vesicles induce the production of anti-LG3 antibodies both in grafted and naïve mice prompted us to further analyze their protein signature. Intriguingly, we observed a strong enrichment for the proteasome core complex specifically in apoptotic exosome-like vesicles (Figures $4 a$ and $4 b$ ). In our proteomics dataset, all the catalytic subunits of the $20 \mathrm{~S}$ proteasome core were identified 
with high confidence in the exosome-like vesicles, while these proteins were absent in apoptotic bodies (Figures $4 a$ and $4 b$ ). Western blot analysis further confirmed the presence of the alpha-3 subunit of the proteasome specifically in exosome-like vesicles (Figure 4a). In contrast, only 4 proteasome-associated proteins were identified in apoptotic bodies, all contained within the 19S regulatory subunit. Immunogold labelling for the $20 \mathrm{~S}$ proteasome identified immunoreactivity in EVs of $30-100 \mathrm{~nm}$ in size (Figure 4c).

Quantitative proteomics based on spectral count indicated comparable levels of all three catalytic subunits containing chymotrypsin-like ( $\beta 5)$, trypsin-like ( $\beta 2)$ and caspase-like ( $\beta 1)$ activities in exosome-like vesicles (Supplementary Table 1). However, we detected several trypsin inhibitors (serpins) in our dataset (Supplementary Table 1). Notably, inter-alpha-trypsin inhibitor heavy chain $\mathrm{H} 2(\mathrm{ITIH} 2)$ is exclusively found in exosome-like vesicles. SERPINE1 (plasminogen activator inhibitor 1) is also enriched in exosome-like vesicles while it is essentially absent in apoptotic bodies. The presence of inhibitors of specific enzymatic activities in the exosome-like vesicles could result in a dynamic modulation of the proteasome activity. Thus, we evaluated whether proteasome activity was present in the various fractions. Consistent with our proteomics findings, we found enhanced caspase-like activity/ug of total proteins in the apoptotic exosome-like vesicle fraction compared to apoptotic bodies and parent cells (Figure 4d). The enhancement of caspase-like activity over other proteolytic functions of the proteasome correlated with the identification of trypsin and chymotrypsin inhibitors in the apoptotic nanovesicle fraction. 
To evaluate whether the presence of active proteasome is a major characteristic of apoptotic exosome-like vesicle, we evaluated if healthy endothelial cells could also secrete membrane vesicles containing active $20 \mathrm{~S}$ proteasome. To avoid contamination of culture medium by membrane vesicles derived from fetal bovine serum (FBS), we generated vesicle free normal medium using FBS spun at 200,000xg before its addition to culture medium (Supplementary figure 14a). Endothelial cells were exposed to normal culture medium for 4 hours. Small particle flow-cytometry demonstrated the presence of exosome-size membrane vesicles in medium conditioned by healthy cells (Supplementary figure 14b). In comparing by western blot equal amounts of membrane vesicles produced by normal or serum starved apoptotic endothelial cells and isolated by centrifugation at $200,000 x \mathrm{x}$, we found that the exosome markers fibronectin, synthenin and TCTP were present in both conditions (Supplementary figure $14 \mathrm{~d}$ ). However, the 20S proteasome core complex and caspase-like proteasome activity were detected exclusively in membrane vesicles released by serum starved apoptotic endothelial cells (Supplementary figure $14 \mathrm{c}$ and d). These results support the notions that active $20 \mathrm{~S}$ proteasome is secreted within exosome-like vesicles specifically under pro-apoptotic serum starved conditions.

We than investigated if the presence of an active proteasomal core complex in apoptotic exosome-like is specific to apoptotic endothelial cell-derived vesicles. To this end we evaluated preparations of apoptotic exosome-like vesicles from medium conditioned by serum starved vascular smooth muscle cells and tubular epithelial cells undergoing 
similar levels of apoptosis as serum starved endothelial cells (Supplementary figure 15a and 15b). Preparations from both cell types highlighted the presence of the $20 \mathrm{~S}$ proteasome core complex by Western blot analysis as well as proteasome activity (Supplementary figure $15 \mathrm{c}$ and $15 \mathrm{~d}$ ), demonstrating that the presence of an active proteasome core complex is not exclusive to endothelial apoptotic exosome-like vesicles.

To investigate whether the activity of the proteasome modulates the release and immunogenic activity of apoptotic exosome-like vesicles, we exposed ECs to bortezomib, a proteasome inhibitor, during serum starvation. Proteasome inhibition neither modulated endothelial apoptosis (Supplementary figure 16) nor prevented the production of EVs but dramatically reduced caspase-like activity in exosome-like vesicles (Figures $4 \mathrm{e}$ and $4 \mathrm{f}$ ). We also performed large-scale proteomics on purified apoptotic exosome-like vesicles from bortezomib- or vehicle-treated apoptotic endothelial cells. Bortezomib treatment strongly modulated the protein content of exosome-like vesicles and increased the number of proteins identified (Figure $4 \mathrm{~g}$ ). Proteasome inhibition resulted in the enrichment of LG3 within exosome-like vesicles suggesting that LG3 is a proteasome substrate (Figure 4h). While 154 proteins were found in control exosome-like vesicles, 337 proteins were identified in bortezomibtreated exosome-like vesicles (Supplementary Table 2). Also, 198 proteins were detected exclusively in exosome-like vesicles from bortezomib-treated cells, suggesting an important role for the proteasome in the degradation of vesicle proteins (Supplementary Table 2). Proteasomal degradation occurs in an ubiquitin-dependent 
manner. We therefore identified ubiquitylated proteins by mass spectrometry. Proteasome inhibition with bortezomib resulted in the accumulation of ubiquitylated proteins in exosome-like vesicles (Figure 4i). Upon bortezomib treatment, 20 ubiquitylation sites on 19 proteins were detected in nanovesicle extracts, while exosome-like vesicles produced in vehicle-treated serum starved cells showed only 5 ubiquitylation sites on 5 proteins (Supplementary Table 3). Collectively these results support the notion that the proteasome, active within apoptotic exosome-like vesicles, regulates the processing of various proteins, including LG3.

Proteasome activity is required for induction of anti-LG3 production and aggravation of allograft inflammation

Since LG3 behaves as a proteasome substrate, we investigated the possibility that the proteasome-activity of apoptotic exosome-like vesicles regulates their capacity to induce the production of anti-LG3 autoantibodies. Both allografted and non-grafted mice were injected with an equal number of apoptotic exosome-like vesicles purified from either bortezomib-treated serum-starved endothelial cells or control vehicle-treated serum-starved endothelial cells. Naïve and transplanted mice exposed to apoptotic exosome-like vesicles from bortezomib-treated cells showed reduced levels of anti-LG3 antibodies when compared to control exosome-like vesicles (Figure 5a). In transplanted mice, neointimal remodelling was similar in mice injected with exosome-like vesicles from bortezomib-treated cells or control apoptotic cells (Figure 5b). Allograft infiltration by CD3+ T cells and CD20+ B cells and C4d deposition however were significantly 
decreased in mice injected with apoptotic exosome-like vesicles from bortezomibtreated cells (Figures $5 b, 5 c$ and $5 d$ ). Collectively, these results suggest that proteasome activity within apoptotic exosome-like vesicles regulates anti-LG3 autoantibody formation and allograft inflammation.

Vascular injury increases proteasome-activity in circulating exosome-like vesicles and triggers anti-LG3 production in vivo

We then evaluated whether this pathway is recapitulated in vivo following vascular injury. We first focussed on renal artery clamping, a model of acute kidney injury (AKI), associated with increased caspase-3 activation within the renal microvasculature (4851). We chose this model because anti-LG3 antibodies have been found in renal disease patients awaiting a kidney transplant and AKI is common in these patients and predicts progressive renal dysfunction (52). In this model, renal function was reduced up to 7 days post-IRI (Figures $6 a$ and $6 b$ ). The $20 S$ proteasome core complex was identified within circulating exosome-like vesicles purified from mouse serum 2 days post-AKI by immunogold-labelling but was absent from exosome-size circulating vesicles purified from normal mice serum (Figure $6 g$ and Supplementary figure 17). Moreover, both proteasome caspase-like activity and LG3 levels increased in exosomelike vesicles preparations after AKI (Figures 6c, 6e and 6f). Anti-LG3 IgG antibodies titers increased significantly 7 and 14 days post-surgery and returned to baseline at 21 days (Figure 6d).

We studied a second model of vascular injury evoked by femoral arteriectomy leading to hind-limb ischemia (Figure 7a), taking into consideration the prevalence of severe 
peripheral vascular disease in the end-stage renal disease population. Again, proteasome caspase-like activity and LG3 levels in circulating exosome-like vesicles increased significantly post-femoral arteriectomy (Figures 7b, 7c and 7d). Anti-LG3 IgG titers also increased significantly at 7 days post-surgery and remained elevated for up to 21 days (Figure 7e). Collectively, these results demonstrate that vascular injury in vivo leads to enhanced caspase-like proteasome activity in circulating exosome-like vesicles followed by the production of anti-LG3 antibodies. 


\section{Discussion}

Apoptosis is classically considered an anti-inflammatory and tolerogenic type of cell death (34). Mounting evidence, however, shows that the presence of autoantibodies reactive with components of apoptotic cells prior to solid organ transplantation predicts reduced allograft survival and risk of rejection $(8,13,18,19,30)$. The present study identifies apoptotic exosome-like vesicles as a novel and immunogenic component of the paracrine apoptotic response. Using electron microscopy, unbiased proteomics and functional studies, we show that nanoscale extracellular vesicles released by apoptotic cells display immune-mediating functions distinct from apoptotic bodies. Apoptotic exosome-like vesicles induce B-cell responses in naïve and transplanted mice leading to the production of autoantibodies. Apoptotic exosome-like vesicles, but not classic apoptotic bodies, aggravate vascular rejection, with increased allograft infiltration by CD3+ T cells and CD20+ B cells, C4d deposition and vascular remodelling. C4d deposition induced by exosome-like vesicles is likely dependent on non-HLA-antibodies, as exosome-like vesicles did not modulate levels of anti-HLA antibodies in rejecting mice and triggered the production of complement-fixing autoantibodies such as antiLG3, known to promote C4d deposition (8).

Our results highlight the complexity of paracrine signals released by apoptotic cells downstream of caspase-3 activation, with the concomitant release of tolerogenic and immunogenic signals. Unbiased comparative proteomic analysis established that different and almost mutually exclusive sets of proteins are present in apoptotic bodies 
and apoptotic exosome-like vesicles, suggesting that their generation requires differential sorting of proteins and that their biogenesis is likely different. While it is well documented that caspase-3 activation regulates the generation of apoptotic bodies by plasma membrane blebbing (53), our results propose an important role for caspase-3 activation in the release of apoptotic exosome-like vesicles. Although the precise molecular components regulating differential sorting of proteins into either apoptotic bodies or exosome-like vesicles is unclear, the present results suggest that distinct molecular pathways activated downstream of activated caspase-3 lead to the production of either apoptotic bodies or apoptotic exosome-like vesicles. Whether nanovesicles are shed from the plasma membrane, generated by exocytosis of Multi Vesicular Bodies (MVB) in a caspase-dependent manner or by another mechanism still remains to be determined. We showed in previous work that serum starvation leads to the accumulation of multivesicular structures (39) but the link between with the biogenesis of apoptotic exosome-like nanovesicles and MVBs requires further investigations. Apoptotic exosome-like vesicles were found to express a protein signature that is both closely related yet distinct from classical exosomes. The presence and absence of different sets of proteins have been suggested as minimal requirements to characterize extracellular vesicles (54). Transmembrane or lipid-bound extracellular proteins (such as CD9, CD63, CD81, integrins, cell adhesion molecules, growth factor receptors, heterotrimeric $G$ proteins, MFG-E8) and cytosolic proteins (TSG-101, annexins, Rabs, syntenin) and the under-representation of endoplasmic reticulum, Golgi, mitochondrial and nuclear proteins are considered characteristic of exosomes (54). Our proteomics data identified integrins (ITGA5, ITGB1), adhesion molecules 
(MCAM), annexins (ANXA1, ANXA2, ANX5) and syntenin in our preparations and confirmed the under-representation of ER, Golgi, mitochondrial and nuclear proteins suggesting that small vesicles released by apoptotic cells display typical characteristics of exosomes. Our results also highlighted some distinctive feature such as release through caspase-3-dependent pathways and the presence of an active proteasome core complex in small vesicles released by apoptotic cells, hence our choice to refer to them as «apoptotic exosome-like» vesicles and not as classical exosomes.

One of the most striking observations of this study is the identification of proteasome activity in endothelial exosome-like vesicles. In eukaryotic cells, proteasomes are highly conserved protease complexes typically composed of the 19S regulatory cap and the $20 S$ proteolytic core. The proteasomal degradation pathway is essential for many cellular processes, including the cell cycle, the regulation of gene expression, and antigenic presentation. In humans, extracellular proteasomes have been found circulating in the plasma of patients suffering from a variety of inflammatory, autoimmune and neoplastic diseases (55-60) and, in various conditions, the concentration of circulating proteasomes correlates with disease activity (61). The presence of circulating proteasome is intriguing since proteasomal subunits do not contain signal sequence for export via the classic secretory pathway, which argues for an alternative secretion mechanism. The release of proteasome in the extracellular milieu as a result of membrane disruption is proposed and cannot be excluded, but the present results and other studies support the existence of regulated mechanisms. The release of active 20S proteasome has also been described recently in EVs originating 
from other cell types such as T cells (shedding microparticles) (62) and mesenchymal stem cells (in exosomes derived from the endosomal compartment) (63). Here we demonstrate that the release of active proteasome within exosome-like vesicles is occurs in association with cell stress and stimulation of apoptotic as healthy endothelial cells do not secrete active proteasome within membrane vesicles. However the secretion of the proteasome core complex in apoptotic vesicles is not specific to endothelial cells as we found the presence and activity of the proteasome in nanovesicles produced by other cell type undergoing apoptosis such as vascular smooth muscle cells and tubular epithelial cells, suggesting that this pathway is likely applicable to various disease states associated with apoptotic cell death.

Our results also show that proteasome activity within apoptotic exosome-like vesicles is one of the key determinants of their immunogenicity. Inhibiting proteasome activity in parent apoptotic endothelial cells did not modulate cell death and the release of apoptotic exosome-like vesicles but completely abrogated all proteasome activity in exosome-like vesicles. Injection of exosome-like vesicles with inhibited proteasome activity elicit significantly less anti-perlecan/LG3 production when injected in naïve mice. Injection of these vesicles in transplanted animals also resulted in reduced levels of anti-LG3 antibodies as well as lower numbers of graft infiltrating $T$ and $B$ cells and reduced C4d deposition. Reduced anti-perlecan/LG3 production occurs in spite of higher levels of perlecan/LG3 in proteasome-inhibited vesicles, demonstrating that failure to trigger an antibody-response is not caused by lower levels of the immunogen. 
Although the detailed characterization of the specific molecular components regulating perlecan/LG3 immunogenicity by the active proteasome core within exosome-like vesicles will require further investigation, our results demonstrate that proteasome activity within these vesicles is central to induction of anti-perlecan/LG3 production in vivo. Bortezomib is currently being used in the treatment of refractory antibodymediated rejection as a B cell-depleting agent based on its pro-apoptotic activity on plasma cells and $\mathrm{B}$ cells $(64,65)$. The present results add further insights into additional pathways of potential importance in the anti-rejection activity of bortezomib. By lowering the immunogenicity of exosome-like vesicles produced at sites of vascular injury, bortezomib could prevent the formation of autoantibodies that further accelerate leukocyte infiltration and complement deposition. The proteasome activity of exosomelike vesicles was, however, dispensable for acceleration of neointima formation. Various factors could explain this finding. The perlecan fragment LG3 per se acts as a promigration factor on mesenchymal stem cells and smooth muscle cells thus promoting their accumulation of sites of vascular injury $(46,47)$ and perlecan fragments have also been reported to bind TLR 2 and 4 (66). Other proteins and mediators present in exosome-like vesicles such as tissue degrading enzymes (metalloproteinases 2 and 9), DAMPs/ TLR ligands (heat shock proteins 75, 10, 71, 70 and fibronectin) and PAI-1 (SERPINE1), could also potentially accelerate the fibroproliferative and inflammatory responses of the vessel wall. Moreover, studying the nucleic acid and lipid content of these exosome-like vesicles might offer additional insights into mechanisms induced by apoptotic exosome-like vesicles. 
Humoral immune responses against perlecan have been found to be associated with rejection in several human transplant cohorts and in animal models of acute and chronic allograft rejection $(8,21,67,68)$. In all these settings, I/R injury have been suspected to play a role in anti-perlecan autoimmune responses. Since anti-perlecan antibodies have been described prior to transplantation in patients without autoimmune conditions (29), we evaluated whether episodes of vascular injury, which are common in renal disease patients, could be associated with increased circulating levels of markers characteristic of apoptotic exosome-like vesicles. Two models of vascular injury in mice led to increased caspase-like activity and LG3 levels in circulating exosome-like vesicles followed by elevated anti-LG3 IgG levels, confirming a close association between vascular and/or tissue injury, release of immunogenic exosome-like vesicles and production of anti-LG3.

Our study has however a number of limitations. First, the biogenesis of apoptotic exosome-like vesicles remains incompletely understood. The abundance of exosomal markers suggests that inward budding of multivesicular bodies could be contributing to their biogenesis, yet how caspase-3 impacts intracellular trafficking and membrane fusion events leading to their release will require further investigation. Second, although we have showed that circulating levels of proteasome-labeled vesicles increase after renal ischemia-reperfusion injury and that proteasome caspase-like activity is increased in these vesicle preparations, we cannot determine precisely the cellular origin of these vesicles. Endothelial cells, vascular smooth muscle cells and tubular epithelial cells all release proteasome-active vesicles when injured in vitro. These cell types are targets of 
injury during ischemia/reperfusion and could all be contributing to the release of apoptotic exosome-like vesicles in vivo. Further studies will be required to identify celltype specific markers of apoptotic exosome-like vesicles. Finally, we have demonstrated that exosome-like vesicles accelerate vascular rejection without increasing the production of donor-specific alloantibodies but rather by triggering the formation of humoral auto-immune pathways. The current study focused primarily on the protein composition of exosome-like vesicles and apoptotic bodies, and enabled the identification of source proteins from which could be derived antigenic peptides. It is also possible that these vesicles may contain small peptides from proteasome degradation that remained elusive to the current proteomics approach. The identification of these peptides and of specific perlecan-derived MHC class I or class II epitopes will require alternate methods that facilitate their recovery and further characterization by MS/MS sequencing to account for the diversity of the corresponding degradation products. This will be the subject of future investigations.

Collectively, these results support the notion that vascular injury in association with renal injury prior to transplantation, leads to increased circulating proteasome activity within exosome-like vesicles fractions, therefore increasing sensitization to autoantigens. By providing novel insights into pathways responsible for the production of autoantibodies prior to transplantation, these results suggest that assessment of proteasome activity within circulating apoptotic exosome-like vesicles before and at the time of transplantation represents new avenues for predicting and controlling 
maladaptive humoral responses to apoptotic cell components that enhance risk of rejection post-transplantation. 


\section{Materials and Methods}

\section{Study design}

The aim of this study was to evaluate the immunogenic potential of the various extracellular structures released by apoptotic endothelial cells. We use large scale proteomics to profile the protein signatures of apoptotic bodies and apoptotic exosomelike vesicles generated in vitro. We then compared the capacity of apoptotic exosomelike vesicles and apoptotic bodies to trigger the production of autoantibodies in transplanted and non-transplanted mice and shape the severity of rejection. The observation that exosome-like vesicles induced the production of anti-LG3 antibodies both in grafted and naïve mice prompted us to further analyze their protein signature. We found a strong enrichment and activity of the $20 \mathrm{~S}$ proteasome core complex in apoptotic exosome-like vesicles by proteomic analyses, immunoblots and electron microscopy. We next investigated, using inhibition of proteasome activity with bortezomib, whether the activity of the proteasome modulates the release and immunogenic activity of apoptotic exosome-like vesicles in vitro and in vivo. Finally, using in vivo models of vascular injury, we evaluated whether the release of proteasome-active exosome-like vesicles occurs in vivo following vascular injury. Group sizes were selected on the basis of our experience with these systems. Mice were ageand sex-matched between groups. Investigators were not blinded when conducting and evaluating the experiments, and no randomization was necessary. No data were excluded from this study. 


\section{Cell culture and conditioned medium preparation}

\section{Endothelial cells}

Murine EC (mEC) were isolated from the aorta of $\mathrm{C} 57 \mathrm{BL} / 6$ mice grown in DMEM low glucose culture media supplemented with endothelial cell growth supplements (ECGS, Alfa Aesar), 10\% FBS (Invitrogen), 10\% new born calf serum (Invitrogen), heparin (6300U, Sandoz), 1\% penicillin-streptomycin and 1\% fungizone. Human umbilical vein endothelial cells (HUVEC) were obtained from Clonetics and cultured in EGM-2MV complete medium (Clonetics). To generate conditioned medium, cells were exposed to serum free medium alone or in the presence of either pan-caspase inhibitor ZVAD-fmk (100 Um; R\&D system), caspase 3 and 7 inhibitor DEVD (50 uM;R\&D systems), proteasome inhibitor bortezomib (100 ug/ml; Stressmarq) or its vehicle DMSO for $4 \mathrm{~h}$ (HUVECS) or $9 \mathrm{~h}$ (mEC). In previous work, we demonstrated that this system leads to the release of active mediators by apoptotic ECs downstream of caspase-3 activation without cell membrane permeabilization (40).

\section{Human proximal tubular epithelial cells}

Human proximal tubular epithelial cells (hTECs) were isolated as previously described from non-diseased nephrectomy samples from patients with renal cell carcinoma (69). Briefly, cortical tissue from kidney samples were incubated with collagenase (Sigma) for $1 \mathrm{~h}$ and passed through a $70 \mathrm{uM}$ cell strainer (Falcon). Cells were washed and resuspended in HPTC media (10\% FBS (Sigma), 1\% penicillin-streptomycin (Sigma), $1 \%$ hormone mix (Sigma), 25ng/mL human EGF (Sigma), 25mM HEPES(Sigma)) then plated on collagen IV coated plates and grown at 37 degrees Celsius in 5\% CO2. HPTC 
were washed and the media was changed the following day. To generate conditioned medium, cells were exposed to serum free medium for $24 \mathrm{~h}$.

Vascular smooth muscle cells

A7R5 cells (rVSMC) (American Type Culture Collection) were cultured in Dulbecco's Modified Eagle Medium: Nutrient Mixture F-12 supplemented with 10\% fetal bovine serum (FBS; Invitrogen). To generate conditioned medium, cells were exposed to serum free medium for $48 \mathrm{~h}$.

\section{Screening for apoptosis by fluorescence microscopy}

Fluorescence microscopy of unfixed/unpermeabilized adherent cells stained with Hoechst $33342 \quad$ (20-(4-ethoxyphenyl)-5-(4-methyl-1-piperazinyl)-2.50-bi-1-1Hbenzimidazole) $(\mathrm{HT})$ and propidium iodide $(\mathrm{PI})$ was performed as previously described (42).

\section{Immunoblotting}

Proteins were extracted, separated by electrophoresis, transferred to nitrocellulose membranes or PVDF (for caspase 3 detection), and probed. Antibodies used for Western blotting were: antibodies against perlecan (Santa Cruz), 20S proteasome alpha 3 (Santa-Cruz), CD63 (Abcam), GM130 (Abcam), tubulin (Calbiochem), Syntenin-1 (Santa Cruz), TCTP (Santa Cruz) and fibronectin (Abcam).

\section{Flow cytometry analyses of EVs}


The flow cytometry analyses were done as previously described in Rousseau et al. (70). In short, fluorescence was used as trigger signal and positive fluorescent events were plotted on an SSC/FSC-PMT graph. The nanovesicle gate of detection was determined based on the acquisition of Sky blue and yellow-green microspheres of 90, 450, 840, 1000 and $3200 \mathrm{~nm}$ (Supplementary Figure 1) and 1000 microspheres were acquired. Conditioned media were labeled for 30 min with V450 probe-conjugated Annexin-V, diluted 1:50, (BD bioscience) at room temperature in the dark. Since annexin-V recognizes PS in a calcium dependent manner, we confirmed its specific recognition of EVs by chelating $\mathrm{Ca}^{2+}$ ions using samples detection in presence of $50 \mu \mathrm{M}$ EDTA as a baseline (70). To process the data quantitatively, a known number of polystyrene microspheres (15 um diameter; Polysciences, PA, USA) were added to each tube as already described (70). To confirm that events detected were genuine extracellular vesicles and not protein aggregates, their sensitivity to $0.05 \%$ Triton $\mathrm{X}-100$ detergent was assessed.

\section{Preparation of fractions enriched in apoptotic bodies or apoptotic exosome-like vesicles}

Serum-free media conditioned by HUVECs were fractionated using sequential centrifugation: a first centrifugation at $1200 \times \mathrm{xg}$ for $15 \mathrm{~min}$ at $4^{\circ} \mathrm{C}$ to pellet cell debris; a second centrifugation at $50000 \times \mathrm{g}$ for $15 \mathrm{~min}$ at $4^{\circ} \mathrm{C}$ to pellet apoptotic bodies and a final ultracentrifugation $\left(200000 \mathrm{xg}\right.$ for $18 \mathrm{~h}$ at $\left.4^{\circ} \mathrm{C}\right)$ to pellet exosome-like vesicles. 
Pellets containing either apoptotic bodies or exosome-like vesicles were resuspended in half the initial volume of conditioned medium. For chronic exposure to the different apoptotic vesicles, a volume of 150ul of either exosome-like vesicles or apoptotic bodies preparation or vehicle (PBS) was injected i.v. (caudal vein) every second day up to 8 injections in the different animal models used (see animal section below).

\section{Proteasome activity assay}

The proteasome activity assay was performed in white 96 wells plates using the Proteasome-GloTM chymotrypsin-like, trypsin-Like and caspase-Like Cell-Based Assays (Promega) in accordance with the manufacturer's instructions using cells lysate (0.4 ug), exosome-like vesicles ( $0.4 \mathrm{ug})$ or apoptotic bodies from HUVEC supernatant $(0.4 \mu \mathrm{g})$ or exosome-like vesicles purified from mouse serum (50ug). Luminescence was measured using a Perkin Elmer Victor 3 V 1420 MultilabelCounter 1420-040 Microplate Reader.

\section{Electron microscopy}

\section{Apoptotic bodies}

The fraction enriched in apoptotic bodies was fixed in $2 \%$ glutaraldehyde- $0.1 \mathrm{M}$ sodium cacodylate, post-fixed in $1 \% \mathrm{OsO}_{4}$, dehydrated in alcohol, processed for flat embedding in Epon 812 and observed at the Zeiss CEM 902 electron microscope, as previously described (71).

Exosome-like vesicles 
Preparations of exosome-like vesicles were fixed in 4\% paraformaldehyde in PBS buffer and were either, contrasted and embedded for whole-mount EM observation or processed for immunolabelling. Fixed exosome-like vesicles $(5 \mathrm{uL})$ were deposit on a Formvar/carbon coated grid for 20 min and washed on drops of PBS at RT. The grid was then directly processed for negative staining or immunolabeling. Negative staining: The grid was transferred onto a drop of $1 \%$ glutaraldehyde for 5 min and washed on several drops of water. Contrasting was performed by transferring the grid onto a drop of $2 \%$ uranyl acetate $-0.075 \mathrm{M}$ oxalate $\mathrm{pH} 7$ solution for 5 min. Finally the grid was transferred onto a drop of $2 \%$ methyl cellulose-4 \% uranyl acetate (9:1) solution for 10 min on ice and excess solution was blotted with Whatman filter paper and air dried. Examination was performed with a Philips CM100 electron microscope. Immunolabeling: The grid was blocked by incubating in 50mM glycine in PBS for 15 min then transferred onto $1 \%$ ovalbumin in PBS for $10 \mathrm{~min}$. The grid was incubated for 30 min on a drop of rabbit anti-LG3 antibody or rabbit anti-proteasome antibody, both diluted at 1:50 in PBS (Santa Cruz Biotechnology, Inc.), and washed on several drops of PBS. Finally, the grid was incubated on a drop of anti-rabbit lgG conjugated to $10 \mathrm{~nm}$ gold particles for 20 min, washed with PBS and processed for negative staining.

\section{Proteomic analysis of purified exosome-like vesicles}

\section{SDS-PAGE and mass spectrometry}

Proteins $(20 \mathrm{ug})$ were separated on a $4-12 \%$ pre-cast NuPAGE gel (Invitrogen). The gel was Coomassie stained, the lanes were cut into pieces, reduced with dithiothreitol (DTT; Sigma Aldrich) and alkylated by Chloroacetamide (Sigma-Aldrich). The gel pieces 
were then digested by trypsin and peptides extracted three times with $90 \%$ acetonitrile $(\mathrm{ACN}) / 0.5 \mathrm{M}$ Urea. Combined extracts were desalted with ZipTips (Millipore), dried and re-suspended in $5 \% \mathrm{ACN}, 0.2 \%$ Formic acid (FA) for mass spectrometry analyses. Peptides were separated on a $150 \mu \mathrm{m}$ ID, $15 \mathrm{~cm}$ reversed phase nano-LC column (Jupiter C18, $3 \mu \mathrm{m}, 300 \AA$, Phenomex) with a loading buffer of $0.2 \%$ formic acid (FA). Peptide elution was achieved by a gradient of $5-40 \%$ ACN in 85 min on an Eksigent 2DnanoLC (Dublin, CA) operating at a flow-rate of $600 \mathrm{~nL} / \mathrm{min}$. The nano-LC was coupled to a LTQ-Orbitrap Elite mass spectrometer (Thermo-Electron) and samples were injected in an interleaved manner. The mass spectrometer was operated in a datadependent acquisition mode with a 1-s survey scan at 120000 resolution, followed by 12 product ion scans (MS/MS) of the most abundant precursors above a threshold of 10,000 counts in the LTQ part of the instrument. CID was performed in the LTQ at $35 \%$ collision energy and an Activation $Q$ of 0.25 . For each mass spectrometry-based proteomics experiment three independent biological replicates were used in triplicates.

\section{Protein Identification and data analysis}

The centroided MS/MS data were merged into single peak-list files (Distiller, v2.4.2.0) and searched with the Mascot search engine v2.3.01 (Matrix Science) against the forward and reversed IPI human v3.54 database. Mascot was searched with a parent ion tolerance of $0.05 \mathrm{Da}$ and a fragment ion mass tolerance of $0.5 \mathrm{Da}$. Carbamidomethylation of cysteine, oxidation of methionione, deamidation and phosphorylation of serine, threonine and tyrosine residues (and ubiquitylation of lysine 
residues (GlyGly) were specified as variable modifications. Proteins were considered identified when they had at least 2 different peptide identifications and the combined score of unique peptide identifications exceeded the score of the first reverseddatabase hit reaching $2 \%$. This resulted in a false-discovery rate of $<2 \%$ on the peptide level. Relative protein abundance was determined using a redundant peptide counting approach (spectral counts). The value of redundant peptide counts from three identification cycles (on three independent biological replicates) was used to generate heatmaps.

\section{Bioinformatics}

Gene Ontology (GO) annotations for cellular component, biological process and molecular function were obtained from the Gene Ontology project using the DAVID Bioinformatics resources (http://david.abcc.ncifcrf.gov/) (72, 73). To identify GO terms that were statistically over represented in our protein list, we used the binomial statistics tool to compare classifications of multiple clusters of lists to a reference list (Homo sapiens total proteome). Only terms that were significantly enriched/depleted with a $p$ value $<0.05$ were used for the analysis.

\section{Animal studies}

Adult C57BI/6 and BALB/c mice (20-22 g) (Charles River, St-Constant, QC) were maintained on a 12-hour light-dark cycle and fed a normal diet ad libitum. All 
experiments on mice were approved by the CRCHUM's Comité Institutionnel de Protection des Animaux (CIPA).

\section{Aorta transplantation procedures}

Mice were anaesthetized using isoflurane $(2 \%)$ by inhalation. Aortic transplantation was erformed as described elsewhere with minor modifications.(74, 75). Briefly, $1 \mathrm{~mL}$ of heparinized saline $(50 \mu \mathrm{L} / \mathrm{mL})$ was injected into the vena cava to flush the aorta. A 6$\mathrm{mm}$ segment of abdominal aorta from below the renal arteries to just above the aortic bifurcation was excised and soaked in ice-cold $0.9 \%$ normal saline. When mentioned, warm ischemia was induced by clamping the aorta for 15 min before excision from the donor. The grafts were then excised and sutured orthotopically with end-to-end anastomoses using 11-0 nylon interrupted sutures.

\section{Renal ischemia reperfusion injury procedures}

Mice are anesthetized with isoflurane and buprenorphin $(0.05 \mathrm{mg} / \mathrm{kg} / \mathrm{SC})$ and placed on a heated surgical table. After a midline incision, the left kidney was exposed and blood supply was interrupted by application of microvascular clamps on the renal artery. After $30 \mathrm{~min}$, the clamps were released, and reperfusion is visualized. The right kidney was than exposed and ligation of ureter and renal blood vessels with 4-0 suture was performed prior to right kidney nephrectomy. After surgery, analgesic was administered (dextrose $2.5 \%, 0.3 \mathrm{~mL}$ ).

\section{Murine ischemic hindlimb model}


Unilateral hindlimb ischemia, a model of persistent vascular injury (76), was surgically induced in mice by femoral arteriectomy as previously described (77). Briefly, the animals were anesthetized with $2 \%$ isoflurane, after which an incision was made in the skin overlying the middle portion of the left hind limb. After ligation of the proximal end of the femoral artery, the distal portion of the saphenous artery was ligated, and the artery and all side branches were dissected free and excised. The skin was closed with a prolene monofilament (6-0) (Johnson \& Johnson).

\section{Isolation of apoptotic exosome-like vesicles from murine serum}

Serum samples collected from mice pre surgery or 2 days post surgery $50 \mu \mathrm{L}$ (to assay proteasome activity) or $25 \mu \mathrm{L}$ (for western blot analyses) were diluted in $12 \mathrm{~mL}$ of RPMI and ultracentrifuged first at $50,000 \mathrm{xg}$ for $15 \mathrm{~min}$ at $4^{\circ} \mathrm{C}$ to pellet cell debris, apoptotic bodies and microparticles and after at $200,000 \mathrm{xg}$ for $18 \mathrm{~h}$ at $4^{\circ} \mathrm{C}$ to pellet exosome-like vesicles. After the last centrifugation, the pellets were resuspended in $2.4 \mu \mathrm{l} / \mu \mathrm{L}$ of original serum sample in either PBS (to assay proteasome activity) or in Laemli buffer (for western blot analyses).

\section{Injection of murine apoptotic endothelial membrane vesicles}

Serum-free media conditioned by $1 \times 10^{4}$ murine endothelial cells were fractionated using sequential centrifugation: a first centrifugation $1200 \times \mathrm{g}$ for $15 \mathrm{~min}$ at $4^{\circ} \mathrm{C}$ to pellet cell debris; a second centrifugation at $50000 \times \mathrm{g}$ for $15 \mathrm{~min}$ at $4^{\circ} \mathrm{C}$ to pellet apoptotic bodies and a final ultracentrifugation $\left(200000 \times \mathrm{g}\right.$ for $18 \mathrm{~h}$ at $\left.4^{\circ} \mathrm{C}\right)$ to pellet exosome-like 
vesicles. Pellets containing either apoptotic bodies or exosome-like vesicles were resuspended in half the initial volume of conditioned medium. Non-grafted or transplanted mice received tail vein (150 ul) IV injections of resuspended preparations every other day during three weeks for a total of 8 doses. In transplanted mice, the first injection occurred 2 days post-transplantation.

\section{Assessment of circulating levels of total IgGs, anti-nuclear antibodies (ANA) and anti-LG3}

Antinuclear antibodies and total lgG levels were assessed using ANA mouse bioassay kits (USBiologicals) and Mouse IgG total Ready-SET-Go kits (Affimetrix), respectively, in accordance with manufacturer's instructions. Anti-LG3 titers were measured with a locally developed ELISA. Recombinant LG3 was produced and purified as previously described (78). Purity of the recovered LG3 protein was assessed by reducing SDSPAGE and Coomassie blue R250 staining. Recombinant mouse LG3 (10 ng/uL) was first coated on 96-well Immulonll HB plates (Thermo Electron), for a total of 1ug per well. Notably, mouse and human LG3 fragments are highly (87\%) homologous at the amino acid level. The sera were diluted (1:250), and $100 \mathrm{uL}$ were added per well. The plates were washed, and bound IgG were detected using horseradish peroxidase coupled with anti-human or anti-mouse IgG (Amersham and Santa-Cruz respectively) or anti-lgG1, IgG2a, IgG2b, IgG3 for IgG subclasses studies(Santa-Cruz). Reactions were

revealed with $100 \mathrm{uL}$ of tetramethylbenzidine substrate (BD Biosciences) and stopped with $50 \mathrm{uL}$ of sulfuric acid $\left(\mathrm{H}_{2} \mathrm{SO}_{4}\right)$. Spectrophotometric analysis was taken at $450 \mathrm{~nm}$ and the results were expressed as optical density. 


\section{Measurement of murine anti-donor IgG}

Sera were diluted 1:100 in FACS buffer and incubated with $1 \times 10^{6}$ BALB/c splenocyte targets for $30 \mathrm{~min}$ at $4^{\circ} \mathrm{C}$. The samples were then washed three times and stained with PE goat anti-mouse IgG (1:100, Alexa 488 anti-mouse CD3e, BD Biosciences) in FACS buffer for $30 \mathrm{~min}$ at $4^{\circ} \mathrm{C}$, in the dark. Samples were run on a flow cytometer (FACScan, BD) and analyzed on computer software (FACS DIVA, Becton-Dickinson). CD3+ parent gate was utilized to avoid nonspecific background signal due to Fc receptor-expressing cells.

Flow cytometry analyses for splenic germinal center B cells and follicular helper T cells

Single-cell suspensions from the spleen were prepared and stained immediately for three-color flow cytometry. The percentage of CD4+PD-1+CXCR5+ Tfh cells among CD4+ cells was determined by flow cytometry using anti-programmed death-1(PD-1) FITC (eBioscience), anti-CXCR5 Biotin (BD biosciences), anti-CD8 APC (Biolegend), anti-CD4 PerCP (BD biosciences), anti-CD62L PE (BD biosciences), anti-CD44 Pacific blue (Biolegend) mAbs and Strep APC Cy7 (BD biosciences). The percentage of B220+GL7+Fas+ GC B cells among B220+ B cells was determined by flow cytometry using anti-B220 APC Cy7 (BD bioscience), anti-GL7 FITC (BD bioscience) and anti-Fas PE (eBioscience) mAbs. 


\section{Immunohistochemistry}

Transplanted and adjacent native aortas were harvested three weeks posttransplantation. Tissues were fixed with $10 \%$ neutral buffered formalin and paraffin embedded according to established methods. Samples were cut into $4 \mu \mathrm{m}$ slices. Immunohistochemistry was assessed on an immunostainer (Discovery XT system, Ventana Medical Systems, Tucson, AZ) in accordance with manufacturer's recommendations. Antigen retrieval was performed with proprietary reagents. When mentioned, samples were stained with haematoxylin and eosin (H\&E). For the detection of $\mathrm{CD} 3+, \mathrm{F} 4 / 80$ + cells and C4d deposition, indirect immunoperoxidase staining was performed using the primary anti-CD3, anti-F4/80 antibody (1:50, AbD Serotec) or antiC4d (1:50, Biomedica), respectively, followed by incubation with specific secondary biotinylated antibodies. Streptavidin horseradish peroxidase and 3,3-diaminobenzidine were used according to the manufacturer's instructions (DABmap detection kit, Ventana Medical Systems). Finally, sections were counterstained with hematoxylin. Digital images of tissues were captured by Leica DMLS microscope and Leica DFC420C camera (Leica Microsystems). Intimal and medial areas grafts were outlined and quantified using a digital image analysis program (ImageJ 1.42q, NIH). The presence of T cells and macrophages in the tissue were quantified by quantification of the number of CD3+ or F4/80+ cells respectively per 6 high power fields per allograft. C4d deposition was quantified using Visiomorph ${ }^{\mathrm{TM}}$ VIS Histoinformatics Software (Olympus)

\section{Statistical analyses}


All data are expressed as Means \pm SEM derived from at least three independent experiments unless otherwise specified. Biological and histological data were compared using $\mathrm{t}$ test. Statistical analyses were performed using Prism 4 (Prism-GraphPad software, Inc). $p$ values of less than 0.05 were considered significant. 


\section{Supplementary Materials}

Supplementary figure 1. Serum deprivation significantly increased caspase-3 activation and the percentage of cells with chromatin condensation in absence of cell membrane permeabilization

Supplementary figure 2. Small particle flow cytometry size calibration

Supplementary figure 3. Characterization of small particles secreted by apoptotic endothelial cells sensitivity to detergent treatment

Supplementary figure 4 . Inhibition of autophagy with bafilomycin does not modulate exosome-like vesicle secretion by serum starved endothelial cells

Supplementary figure 5. Electron micrographs of apoptotic bodies released by serumstarved HUVECs and isolated by sequential ultracentrifugation

Supplementary figure 6 . Electron micrographs of exosome-like vesicles released by serum-starved HUVECs and isolated by sequential ultracentrifugation. 
Supplementary figure 7. Gene ontology analysis of cellular components and biological processes for proteins unique to exosome-like vesicles and apoptotic bodies.

Supplementary figure 8. Characterization of the presence of classical exosomal markers in the apoptotic exosome-like vesicles proteome

Supplementary figure 9. Redundant peptide count of Perlecan in exosome-like vesicles and apoptotic bodies.

Supplementary figure 10. Injecting equal amounts of proteins from preparations of apoptotic bodies and preparations of apoptotic exosome-like vesicles fail to unmask immunogenic activity in apoptotic bodies.

Supplementary figure 11. Apoptotic exosome-like vesicles, unlike apoptotic bodies, favor $B$ cell responses and autoimmunity

Supplementary figure 12. Intima/media ratio in murine allografts 3,6 or 9 weeks postaortic transplantation. 
Supplementary figure 13. Characterization of anti-LG3 IgG subclasses

Supplementary figure 14. Healthy endothelial cells secrete exosome-size vesicles lacking caspase-like proteasome activity

Supplementary figure 15. Apoptotic vascular smooth muscle cells and tubular epithelial cells also secrete active $20 \mathrm{~S}$ proteasome in exosome-size extracellular vesicles

Supplementary figure 16 . Bortezomib treatment does not affect cell death levels

Supplementary Figure 17. The proteasome is not detected in serum membrane vesicles isolated pre surgery.

Supplementary table1. Quantitative proteomics identifications based on spectral counts in nanovesicles and apoptotic bodies from serum starved apoptotic HUVECS cells.

Supplementary table 2. Quantitative proteomics identifications based on spectral counts in nanovesicles from serum starved apoptotic HUVECS cells treated with proteasome inhibitor bortezomib or vehicle

Supplementary table 3. Ubiquitylated proteins identification by mass spectrometry in nanovesicles from serum starved HUVECS cells treated with proteasome inhibitor bortezomib or vehicle 


\section{Data S1: Source data, unedited gels}

\section{Data S2: Source data, flow cytometry gating}

\section{References}

1. H. de Kort, M. Willicombe, P. Brookes, K. M. Dominy, E. Santos-Nunez, J. W. Galliford, K. Chan, D. Taube, A. G. McLean, H. T. Cook, C. Roufosse, Microcirculation inflammation associates with outcome in renal transplant patients with de novo donor-specific antibodies. Am J Transplant 13, 485-492 (2013).

2. L. D. Cornell, R. N. Smith, R. B. Colvin, Kidney transplantation: mechanisms of rejection and acceptance. Annu Rev Pathol 3, 189-220 (2008).

3. B. Sis, G. Einecke, J. Chang, L. G. Hidalgo, M. Mengel, B. Kaplan, P. F. Halloran, Cluster analysis of lesions in nonselected kidney transplant biopsies: microcirculation changes, tubulointerstitial inflammation and scarring. Am J Transplant 10, 421-430 (2010).

4. B. Sis, G. S. Jhangri, S. Bunnag, K. Allanach, B. Kaplan, P. F. Halloran, Endothelial gene expression in kidney transplants with alloantibody indicates antibody-mediated damage despite lack of C4d staining. Am J Transplant 9, 2312-2323 (2009).

5. G. Einecke, B. Sis, J. Reeve, M. Mengel, P. M. Campbell, L. G. Hidalgo, B. Kaplan, P. F. Halloran, Antibody-mediated microcirculation injury is the major cause of late kidney transplant failure. Am J Transplant 9, 2520-2531 (2009).

6. A. N. Babu, T. Murakawa, J. M. Thurman, E. J. Miller, P. M. Henson, M. R. Zamora, N. F. Voelkel, M. R. Nicolls, Microvascular destruction identifies murine allografts that cannot be rescued from airway fibrosis. J Clin Invest 117, 3774-3785 (2007).

7. Y. Ishii, T. Sawada, K. Kubota, S. Fuchinoue, S. Teraoka, A. Shimizu, Injury and progressive loss of peritubular capillaries in the development of chronic allograft nephropathy. Kidney Int 67, 321-332 (2005).

8. H. Cardinal, M. Dieude, N. Brassard, S. Qi, N. Patey, M. Soulez, D. Beillevaire, F. Echeverry, C. Daniel, Y. Durocher, F. Madore, M. J. Hebert, Antiperlecan antibodies are novel accelerators of immune-mediated vascular injury. Am J Transplant 13, 861-874 (2013).

9. H. Regele, Non-HLA antibodies in kidney allograft rejection: convincing concept in need of further evidence. Kidney Int 79, 583-586 (2011).

10. D. Saini, J. Weber, S. Ramachandran, D. Phelan, V. Tiriveedhi, M. Liu, N. Steward, A. Aloush, R. Hachem, E. Trulock, B. Meyers, G. A. Patterson, T. Mohanakumar, Alloimmunity-induced autoimmunity as a potential mechanism in the pathogenesis of chronic rejection of human lung allografts. J Heart Lung Transplant 30, 624-631 (2011).

11. V. Carter, B. K. Shenton, B. Jaques, D. Turner, D. Talbot, A. Gupta, C. E. Chapman, C. J. Matthews, G. Cavanagh, Vimentin antibodies: a non-HLA antibody as a potential risk factor in renal transplantation. Transplant Proc 37, 654-657 (2005).

12. S. Jurcevic, M. E. Ainsworth, A. Pomerance, J. D. Smith, D. R. Robinson, M. J. Dunn, M. H. Yacoub, M. L. Rose, Antivimentin antibodies are an independent predictor of transplant-associated coronary artery disease after cardiac transplantation. Transplantation 71, 886-892 (2001).

13. B. Mahesh, H. S. Leong, A. McCormack, P. Sarathchandra, A. Holder, M. L. Rose, Autoantibodies to vimentin cause accelerated rejection of cardiac allografts. Am J Pathol 170, 1415-1427 (2007).

14. B. Mahesh, H. S. Leong, K. S. Nair, A. McCormack, P. Sarathchandra, M. L. Rose, Autoimmunity to vimentin potentiates graft vasculopathy in murine cardiac allografts. Transplantation 90, 4-13 (2010).

15. D. Dragun, Humoral responses directed against non-human leukocyte antigens in solid-organ transplantation. Transplantation 86, 1019-1025 (2008).

16. D. Dragun, B. Hegner, Non-HLA antibodies post-transplantation: clinical relevance and treatment in solid organ transplantation. Contrib Nephrol 162, 129-139 (2009).

17. D. Dragun, D. N. Muller, J. H. Brasen, L. Fritsche, M. Nieminen-Kelha, R. Dechend, U. Kintscher, B. Rudolph, J. Hoebeke, D. Eckert, I. Mazak, R. Plehm, C. Schonemann, T. Unger, K. Budde, H. H. Neumayer, F. C. Luft, G. Wallukat, Angiotensin II type 1-receptor activating antibodies in renal-allograft rejection. N Engl J Med 352, 558-569 (2005). 
18. L. Li, P. Wadia, R. Chen, N. Kambham, M. Naesens, T. K. Sigdel, D. B. Miklos, M. M. Sarwal, A. J. Butte, Identifying compartment-specific non-HLA targets after renal transplantation by integrating transcriptome and "antibodyome" measures. Proc Natl Acad Sci U S A 106, 4148-4153 (2009).

19. T. K. Sigdel, L. Li, T. Q. Tran, P. Khatri, M. Naesens, P. Sansanwal, H. Dai, S. C. Hsieh, M. M. Sarwal, Non-HLA antibodies to immunogenic epitopes predict the evolution of chronic renal allograft injury. $J$ Am Soc Nephrol 23, 750-763 (2012).

20. S. Kalache, R. Dinavahi, S. Pinney, A. Mehrotra, M. W. Cunningham, P. S. Heeger, Anticardiac myosin immunity and chronic allograft vasculopathy in heart transplant recipients. J Immunol 187, 1023-1030 (2011).

21. S. A. Joosten, Y. W. Sijpkens, V. van Ham, L. A. Trouw, J. van der Vlag, B. van den Heuvel, C. van Kooten, L. C. Paul, Antibody response against the glomerular basement membrane protein agrin in patients with transplant glomerulopathy. Am J Transplant 5, 383-393 (2005).

22. M. L. Rose, Role of anti-vimentin antibodies in allograft rejection. Hum Immunol 74, 1459-1462 (2013).

23. A. T. Linke, B. Marchant, P. Marsh, G. Frampton, J. Murphy, M. L. Rose, Screening of a HUVEC cDNA library with transplant-associated coronary artery disease sera identifies RPL7 as a candidate autoantigen associated with this disease. Clin Exp Immunol 126, 173-179 (2001).

24. F. Porcheray, J. DeVito, B. Y. Yeap, L. Xue, I. Dargon, R. Paine, T. C. Girouard, S. L. Saidman, R. B. Colvin, W. Wong, E. Zorn, Chronic humoral rejection of human kidney allografts associates with broad autoantibody responses. Transplantation 89, 1239-1246 (2010).

25. R. S. Warraich, A. Pomerance, A. Stanley, N. R. Banner, M. J. Dunn, M. H. Yacoub, Cardiac myosin autoantibodies and acute rejection after heart transplantation in patients with dilated cardiomyopathy. Transplantation 69, 1609-1617 (2000).

26. N. Angaswamy, C. Klein, V. Tiriveedhi, J. Gaut, S. Anwar, A. Rossi, D. Phelan, J. R. Wellen, S. Shenoy, W. C. Chapman, T. Mohanakumar, Immune responses to collagen-IV and fibronectin in renal transplant recipients with transplant glomerulopathy. Am J Transplant 14, 685-693 (2014).

27. A. Bharat, D. Saini, N. Steward, R. Hachem, E. P. Trulock, G. A. Patterson, B. F. Meyers, T. Mohanakumar, Antibodies to self-antigens predispose to primary lung allograft dysfunction and chronic rejection. Ann Thorac Surg 90, 1094-1101 (2010).

28. V. Subramanian, S. Ramachandran, B. Banan, A. Bharat, X. Wang, N. Benshoff, D. Kreisel, A. E. Gelman, T. Mohanakumar, Immune response to tissue-restricted self-antigens induces airway inflammation and fibrosis following murine lung transplantation. Am J Transplant 14, 2359-2366 (2014).

29. G. Honger, H. Cardinal, M. Dieude, A. Buser, I. Hosli, D. Dragun, M. J. Hebert, S. Schaub, Human pregnancy and generation of anti-angiotensin receptor and anti-perlecan antibodies. Transpl Int 27, 467474 (2014).

30. B. Gao, C. Moore, F. Porcheray, C. Rong, C. Abidoglu, J. DeVito, R. Paine, T. C. Girouard, S. L. Saidman, D. Schoenfeld, B. Levin, W. Wong, N. Elias, C. Schuetz, I. Rosales, Y. Fu, E. Zorn, Pretransplant IgG reactivity to apoptotic cells correlates with late kidney allograft loss. Am J Transplant 14, 1581-1591 (2014).

31. F. Porcheray, J. W. Fraser, B. Gao, A. McColl, J. DeVito, I. Dargon, Y. Helou, W. Wong, T. C. Girouard, S. L. Saidman, R. B. Colvin, A. Palmisano, U. Maggiore, A. Vaglio, R. N. Smith, E. Zorn, Polyreactive antibodies developing amidst humoral rejection of human kidney grafts bind apoptotic cells and activate complement. Am J Transplant 13, 2590-2600 (2013).

32. J. F. Cailhier, I. Sirois, P. Laplante, S. Lepage, M. A. Raymond, N. Brassard, A. Prat, R. V. Iozzo, A. V. Pshezhetsky, M. J. Hebert, Caspase-3 activation triggers extracellular cathepsin L release and endorepellin proteolysis. J Biol Chem 283, $27220-27229$ (2008).

33. P. Laplante, M. A. Raymond, A. Labelle, J. Abe, R. V. Iozzo, M. J. Hebert, Perlecan proteolysis induces an alpha2beta1 integrin- and Src family kinase-dependent anti-apoptotic pathway in fibroblasts in the absence of focal adhesion kinase activation. J Biol Chem 281, 30383-30392 (2006).

34. I. K. Poon, C. D. Lucas, A. G. Rossi, K. S. Ravichandran, Apoptotic cell clearance: basic biology and therapeutic potential. Nat Rev Immunol 14, 166-180 (2014).

35. D. R. Getts, D. P. McCarthy, S. D. Miller, Exploiting apoptosis for therapeutic tolerance induction. J Immunol 191, 5341-5346 (2013).

36. K. S. Ravichandran, U. Lorenz, Engulfment of apoptotic cells: signals for a good meal. Nat Rev Immunol 7, 964-974 (2007). 
37. L. A. Casciola-Rosen, G. Anhalt, A. Rosen, Autoantigens targeted in systemic lupus erythematosus are clustered in two populations of surface structures on apoptotic keratinocytes. J Exp Med 179, 1317-1330 (1994).

38. P. D. Robbins, A. E. Morelli, Regulation of immune responses by extracellular vesicles. Nat Rev Immunol 14, 195-208 (2014).

39. I. Sirois, J. Groleau, N. Pallet, N. Brassard, K. Hamelin, I. Londono, A. V. Pshezhetsky, M. Bendayan, M. J. Hebert, Caspase activation regulates the extracellular export of autophagic vacuoles. Autophagy 8, 927937 (2012).

40. I. Sirois, M. A. Raymond, N. Brassard, J. F. Cailhier, M. Fedjaev, K. Hamelin, I. Londono, M. Bendayan, A. V. Pshezhetsky, M. J. Hebert, Caspase-3-dependent export of TCTP: a novel pathway for antiapoptotic intercellular communication. Cell Death Differ 18, 549-562 (2011).

41. E. I. Buzas, B. Gyorgy, G. Nagy, A. Falus, S. Gay, Emerging role of extracellular vesicles in inflammatory diseases. Nat Rev Rheumatol 10, 356-364 (2014).

42. N. Pallet, I. Sirois, C. Bell, L. A. Hanafi, K. Hamelin, M. Dieude, C. Rondeau, P. Thibault, M. Desjardins, M. J. Hebert, A comprehensive characterization of membrane vesicles released by autophagic human endothelial cells. Proteomics 13, 1108-1120 (2013).

43. N. Cloutier, S. Tan, L. H. Boudreau, C. Cramb, R. Subbaiah, L. Lahey, A. Albert, R. Shnayder, R. Gobezie, P. A. Nigrovic, R. W. Farndale, W. H. Robinson, A. Brisson, D. M. Lee, E. Boilard, The exposure of autoantigens by microparticles underlies the formation of potent inflammatory components: the microparticle-associated immune complexes. EMBO Mol Med 5, 235-249 (2013).

44. L. H. Boudreau, A. C. Duchez, N. Cloutier, D. Soulet, N. Martin, J. Bollinger, A. Pare, M. Rousseau, G. S. Naika, T. Levesque, C. Laflamme, G. Marcoux, G. Lambeau, R. W. Farndale, M. Pouliot, H. HamzehCognasse, F. Cognasse, O. Garraud, P. A. Nigrovic, H. Guderley, S. Lacroix, L. Thibault, J. W. Semple, M. H. Gelb, E. Boilard, Platelets release mitochondria serving as substrate for bactericidal group IIA-secreted phospholipase A2 to promote inflammation. Blood 124, 2173-2183 (2014).

45. O. T. O'Riordan E, Mendelev N, Patschan D, Kemp R, Chander P, Hu R, Hao G, Gross S, Iozzo R, Delaney V, Goligorsky M, Urinary proteomic analysis of chronic allograft nephropathy. Proteomics Clin. Appl. 2, 1025-1035 (2008).

46. M. Soulez, E. A. Pilon, M. Dieude, H. Cardinal, N. Brassard, S. Qi, S. J. Wu, Y. Durocher, F. Madore, C. Perreault, M. J. Hebert, The perlecan fragment LG3 is a novel regulator of obliterative remodeling associated with allograft vascular rejection. Circ Res 110, 94-104 (2012).

47. E. A. Pilon, M. Dieude, S. Qi, K. Hamelin, L. Pomerleau, D. Beillevaire, Y. Durocher, M. Zutter, D. Coutu, C. Perreault, M. J. Hebert, The perlecan fragment LG3 regulates homing of mesenchymal stem cells and neointima formation during vascular rejection. Am J Transplant 15, 1205-1218 (2015).

48. D. P. Basile, The endothelial cell in ischemic acute kidney injury: implications for acute and chronic function. Kidney Int 72, 151-156 (2007).

49. D. P. Basile, J. L. Friedrich, J. Spahic, N. Knipe, H. Mang, E. C. Leonard, S. Changizi-Ashtiyani, R. L. Bacallao, B. A. Molitoris, T. A. Sutton, Impaired endothelial proliferation and mesenchymal transition contribute to vascular rarefaction following acute kidney injury. Am J Physiol Renal Physiol 300, F721-733 (2011).

50. D. P. Basile, M. C. Yoder, Renal endothelial dysfunction in acute kidney ischemia reperfusion injury. Cardiovasc Hematol Disord Drug Targets 14, 3-14 (2014).

51. A. A. Sharfuddin, B. A. Molitoris, Pathophysiology of ischemic acute kidney injury. Nat Rev Nephrol 7, 189-200 (2011).

52. B. A. Molitoris, Therapeutic translation in acute kidney injury: the epithelial/endothelial axis. J Clin Invest 124, 2355-2363 (2014).

53. B. Gyorgy, T. G. Szabo, M. Pasztoi, Z. Pal, P. Misjak, B. Aradi, V. Laszlo, E. Pallinger, E. Pap, A. Kittel, G. Nagy, A. Falus, E. I. Buzas, Membrane vesicles, current state-of-the-art: emerging role of extracellular vesicles. Cell Mol Life Sci 68, 2667-2688 (2011).

54. J. Lotvall, A. F. Hill, F. Hochberg, E. I. Buzas, D. Di Vizio, C. Gardiner, Y. S. Gho, I. V. Kurochkin, S. Mathivanan, P. Quesenberry, S. Sahoo, H. Tahara, M. H. Wauben, K. W. Witwer, C. Thery, Minimal experimental requirements for definition of extracellular vesicles and their functions: a position statement from the International Society for Extracellular Vesicles. J Extracell Vesicles 3, 26913 (2014).

55. M. Majetschak, M. Perez, L. T. Sorell, J. Lam, M. E. Maldonado, R. W. Hoffman, Circulating $20 \mathrm{~S}$ proteasome levels in patients with mixed connective tissue disease and systemic lupus erythematosus. Clin Vaccine Immunol 15, 1489-1493 (2008). 
56. L. Henry, T. Lavabre-Bertrand, T. Douche, S. Uttenweiler-Joseph, P. Fabbro-Peray, B. Monsarrat, J. Martinez, L. Meunier, P. E. Stoebner, Diagnostic value and prognostic significance of plasmatic proteasome level in patients with melanoma. Exp Dermatol 19, 1054-1059 (2010).

57. C. Jakob, K. Egerer, P. Liebisch, S. Turkmen, I. Zavrski, U. Kuckelkorn, U. Heider, M. Kaiser, C. Fleissner, J. Sterz, L. Kleeberg, E. Feist, G. R. Burmester, P. M. Kloetzel, O. Sezer, Circulating proteasome levels are an independent prognostic factor for survival in multiple myeloma. Blood 109, 2100-2105 (2007).

58. L. Henry, T. Lavabre-Bertrand, L. Vercambre, J. Ramos, S. Carillo, I. Guiraud, P. Pouderoux, M. Bismuth, J. C. Valats, C. Demattei, Y. Duny, I. Chaze, N. Funakoshi, J. P. Bureau, J. P. Daures, P. Blanc, Plasma proteasome level is a reliable early marker of malignant transformation of liver cirrhosis. Gut 58, 833-838 (2009).

59. M. Wada, M. Kosaka, S. Saito, T. Sano, K. Tanaka, A. Ichihara, Serum concentration and localization in tumor cells of proteasomes in patients with hematologic malignancy and their pathophysiologic significance. J Lab Clin Med 121, 215-223 (1993).

60. M. Heubner, P. Wimberger, B. Dahlmann, S. Kasimir-Bauer, R. Kimmig, J. Peters, J. Wohlschlaeger, S. U. Sixt, The prognostic impact of circulating proteasome concentrations in patients with epithelial ovarian cancer. Gynecol Oncol 120, 233-238 (2011).

61. S. U. Sixt, B. Dahlmann, Extracellular, circulating proteasomes and ubiquitin - incidence and relevance. Biochim Biophys Acta 1782, 817-823 (2008).

62. I. Bochmann, F. Ebstein, A. Lehmann, J. Wohlschlaeger, S. U. Sixt, P. M. Kloetzel, B. Dahlmann, T lymphocytes export proteasomes by way of microparticles: a possible mechanism for generation of extracellular proteasomes. J Cell Mol Med 18, 59-68 (2014).

63. R. C. Lai, S. S. Tan, B. J. Teh, S. K. Sze, F. Arslan, D. P. de Kleijn, A. Choo, S. K. Lim, Proteolytic Potential of the MSC Exosome Proteome: Implications for an Exosome-Mediated Delivery of Therapeutic Proteasome. Int J Proteomics 2012, 971907 (2012).

64. R. C. Walsh, R. R. Alloway, A. L. Girnita, E. S. Woodle, Proteasome inhibitor-based therapy for antibodymediated rejection. Kidney Int 81, 1067-1074 (2012).

65. A. Mulder, S. Heidt, M. Vergunst, D. L. Roelen, F. H. Claas, Proteasome inhibition profoundly affects activated human B cells. Transplantation 95, 1331-1337 (2013).

66. L. Schaefer, Complexity of danger: the diverse nature of damage-associated molecular patterns. J Biol Chem 289, 35237-35245 (2014).

67. S. A. Joosten, M. G. van Dixhoorn, M. C. Borrias, H. Benediktsson, P. A. van Veelen, C. van Kooten, L. C. Paul, Antibody response against perlecan and collagen types IV and VI in chronic renal allograft rejection in the rat. Am J Pathol 160, 1301-1310 (2002).

68. S. A. Joosten, C. van Kooten, Non-HLA humoral immunity and chronic kidney-graft loss. Lancet 365, 1522-1523 (2005).

69. A. Vilaysane, J. Chun, M. E. Seamone, W. Wang, R. Chin, S. Hirota, Y. Li, S. A. Clark, J. Tschopp, K. Trpkov, B. R. Hemmelgarn, P. L. Beck, D. A. Muruve, The NLRP3 inflammasome promotes renal inflammation and contributes to CKD. J Am Soc Nephrol 21, 1732-1744 (2010).

70. M. Rousseau, C. Belleannee, A. C. Duchez, N. Cloutier, T. Levesque, F. Jacques, J. Perron, P. A. Nigrovic, M. Dieude, M. J. Hebert, M. H. Gelb, E. Boilard, Detection and quantification of microparticles from different cellular lineages using flow cytometry. Evaluation of the impact of secreted phospholipase A2 on microparticle assessment. PLoS One 10, e0116812 (2015).

71. L. English, M. Chemali, J. Duron, C. Rondeau, A. Laplante, D. Gingras, D. Alexander, D. Leib, C. Norbury, R. Lippe, M. Desjardins, Autophagy enhances the presentation of endogenous viral antigens on MHC class I molecules during HSV-1 infection. Nat Immunol 10, 480-487 (2009).

72. B. T. a. L. Sherman, R.A., Bioinformatics enrichment tools: Path towards the comprehensive functional analysis of large gene lists. Nucleic Acids Research 37, 1-13 (2009).

73. B. T. S. Da Wei Huang, and Lempicki, R. A., Systematic and integrative analysis of large gene lists using DAVID bioinformatics resources. . Nature protocols 4, 44-57 (2008).

74. J. Koulack, V. C. McAlister, C. A. Giacomantonio, H. Bitter-Suermann, A. S. MacDonald, T. D. Lee, Development of a mouse aortic transplant model of chronic rejection. Microsurgery 16, 110-113 (1995).

75. K. Shimizu, S. Sugiyama, M. Aikawa, Y. Fukumoto, E. Rabkin, P. Libby, R. N. Mitchell, Host bonemarrow cells are a source of donor intimal smooth- muscle-like cells in murine aortic transplant arteriopathy. Nat Med 7, 738-741 (2001). 
76. S. H. Lee, J. H. Lee, Y. S. Han, J. M. Ryu, Y. M. Yoon, H. J. Han, Hypoxia accelerates vascular repair of endothelial colony-forming cells on ischemic injury via STAT3-BCL3 axis. Stem Cell Res Ther 6, 139 (2015).

77. J. Turgeon, S. Dussault, F. Maingrette, J. Groleau, P. Haddad, G. Perez, A. Rivard, Fish oil-enriched diet protects against ischemia by improving angiogenesis, endothelial progenitor cell function and postnatal neovascularization. Atherosclerosis 229, 295-303 (2013).

78. M. Soulez, I. Sirois, N. Brassard, M. A. Raymond, F. Nicodeme, N. Noiseux, Y. Durocher, A. V. Pshezhetsky, M. J. Hebert, Epidermal growth factor and perlecan fragments produced by apoptotic endothelial cells co-ordinately activate ERK1/2-dependent antiapoptotic pathways in mesenchymal stem cells. Stem Cells 28, 810-820 (2010). 
Acknowledgements We wish to thank Melissa Hénault-Rondeau, Suzanne Morissette and Francine Marsan for their work with the Université de Montréal Renal Transplant Biobank, Yves Durocher for providing recombinant LG3, IRIC histology platform and CRCHUM cytometry and microscopy platforms.

Funding: The authors acknowledge support from the Canadian National Transplantation Research Program (CNTRP) (MD, JFC, CP, EB, PT, MJH), the Canadian Institutes of Health Research (CIHR) (MOP-15447) (MJH), the Kidney Foundation of Canada (MJH), CCSRI grant (PT), NSERC grant 311598 (PT) and the Natural Sciences and Engineering Research Council of Canada grant 386598 (EB). MJH is the holder of the Shire Chair in Nephrology, Transplantation and Renal Regeneration of Université de Montréal. We thank the J.-L. Lévesque Foundation for renewed support. The Institute for Research in Immunology and Cancer (IRIC) receives infrastructure support from the Canadian Center of Excellence in Commercialization and Research, the Canadian Foundation for Innovation, and the Fonds de recherche du Québec - Santé (FRQS). EB is a recipient of a Canadian Institutes of Health Research new investigator award. MR was supported by a grant from NSERC. NP received a fellowship grant from Universite de Montreal Nephrology Consortium.

Author contributions: $\mathrm{MD}, \mathrm{CB}, \mathrm{EB}, \mathrm{PT}$ and $\mathrm{MJH}$ develop concepts, designed the experiments, and wrote the manuscript. MD, CB, JT, DB, LP, BY, KH, SQ, AL, NP, CB, ACD, TL, WD, MR, CR, DG and EB performed the experiments and analyzed the data. $\mathrm{HC}$ was the consultant for the statistical analyses. JFC, MR, AR, DM, HC, CP, MD, EB analyzed the data, gave technical support and conceptual advices and edited the manuscript. 


\title{
Data and materials availability: The proteomic data for this study are deposited in
}

\author{
exocarta and vesiclepedia databases.
}

Figure 1: Apoptotic endothelial cells secrete two discrete types of extracellular vesicles, apoptotic bodies and exosome-like vesicles, downstream of caspase- 3 activation.

a) and b) Left panels: Representative FSC-PMT/SSC plots of the extracellular vesicles detected with fluorochromeconjugated annexin-V in the supernatant of serum-starved HUVECs exposed to vehicle, DEVD or ZVAD (a) or mECs from WT or Casp-3 KO mice (b). Two major subpopulations, named exosome-like vesicles (exo-like) and apoptotic bodies (apo bodies), are observed and

gated. Triton panels represent annexin- $V_{+}$events detected in the supernatant after treatment with triton $0.05 \%$ (a) or mECs (b). Right panel: Flow cytometric quantifications of Annexin-V+ apoptotic bodies (white bars) and exosome-like vesicles (black bars) detected in the supernatant of HUVECs (a) or mECs (b). c) Electron micrographs of apoptotic bodies (left panel) and exosome-like vesicles (right panel) released by serumstarved HUVECs and isolated by sequential ultracentrifugation. d) Heatmap representation of protein abundance in isolated exosome-like vesicles and apoptotic bodies. Exosome-like vesicles and apoptotic bodies are characterized by distinct protein patterns. Perlecan is enriched in exosome-like vesicles, while several histones are unique to or enriched in apoptotic bodies. $\mathrm{n} \geq 6$ for each condition. Data are pooled from 3 independent experiments. mean \pm SEM, $\mathrm{t}$-test.

Figure 2. Apoptotic exosome-like vesicles are specifically enriched in Perlecan-LG3 fragment and trigger the production of anti-LG3 autoantibodies

a) Sequence of the LG3 fragment and representation of identified peptides (red) in exosome-like vesicles and apoptotic bodies. The sequence coverage of LG3 is higher in exosome-like vesicles (59\%) vs. apoptotic bodies $(28 \%)$. b) Electron micrographs showing immunogold labeling for LG3 in exosome-like vesicles from serum starved HUVECs. Data are pooled from 3 independent experiments. c)

Representative immunoblot and densitometry analysis for LG3 in exosome-like vesicles or apoptotic bodies purified from serum starved apoptotic HUVECs incubated with the pan-caspase inhibitor Z-VAD or vehicle. d) Representative immunoblot and densitometry analysis for LG3 in exosome-like vesicles or apoptotic bodies purified from serum starved apoptotic mECs from WT of Caspase-3 KO mice. e) Anti-LG3 IgG titers in sera from WT mice after 3 weeks of i.v. injections with vehicle, exosome-like vesicles or apoptotic bodies purified from serum starved murine endothelial cells. $n \geq 6$ for each condition, mean \pm SEM, t-test.

Figure 3. Apoptotic exosome-like vesicles aggravate vascular rejection.

a) Anti-LG3 IgG titers in sera harvested 3 weeks post-surgery from allografted mice injected with vehicle, exosomelike vesicles or apoptotic bodies for 3 weeks post-transplantation. b) H\&E, CD3, CD20 and C4d stained aortic allograft sections from mice injected with vehicle, exosome-like vesicles or apoptotic bodies for 3 weeks posttransplantation (top panels magnification: $5 X$, lower panels magnification: 20X). c) Intima/media ratio in allografts from mice injected with vehicle, exosome-like vesicles or apoptotic bodies for 3 weeks post-transplantation. d) Mean number of CD3 positive cells (T cells) per high power field in allografts from mice injected with vehicle, exosome-like vesicles or apoptotic bodies for 3 weeks posttransplantation. e) Mean number of CD20 positive cells (B cells) per high power field in allografts from mice injected with vehicle, exosome-like vesicles or apoptotic bodies for 3 weeks posttransplantation. f) C4d deposition in allografts from mice injected with vehicle, exosome-like vesicles or apoptotic bodies for 3 weeks posttransplantation. $\mathbf{g}$ ) Donor specific antibodies (DSA) levels in sera harvested 3 weeks post-surgery from allografted mice injected with vehicle, exosome-like vesicles or apoptotic bodies for 3 weeks post-transplantation. $n \geq 8$ for each condition. Data are pooled from 3 independent experiments. mean \pm SEM, comparison with vehicle, t-test.

Figure 4. The proteasome, active within apoptotic exosome-like vesicles, regulates the processing of various proteins, including LG3.

a) Heatmap representation of proteasome protein abundance in exosome-like vesicles and apoptotic bodies. Lower panel: Western blot analysis for alpha 3 proteasome subunit in exosome-like vesicles (exo-like) and apoptotic bodies (apo bodies). b) Schematic representation of the proteins detected specifically in exosome-like vesicles (in red) and in apoptotic bodies (black). The complete proteasome $20 \mathrm{~S}$ core complex is found in exosome-like vesicles while only regulatory subunits are detected in apoptotic bodies. c) Electron micrographs showing immunogold labeling of alpha 3 proteasome subunit in exosome-like vesicles from serum starved HUVECs. d) Quantification of proteasome chymotrypsin, trypsin and caspase-like proteolytic activities in serum starved HUVECs (Grey bars), exosome-like vesicles (Black bars) and apoptotic bodies (White bars). $n \geq 5$ for each condition. mean \pm SEM, comparison with cells e) Proteasome chymotrypsin, trypsin and caspase-like proteolytic activity quantification in exosome-like vesicles from serum starved HUVECS cells treated with the proteasome inhibitor bortezomib or its vehicle. mean \pm SEM, comparison with vehicle f) Quantification, by flow cytometry, of Annexin-V+EVs secreted by serum starved HUVEC 
treated with the proteasome inhibitor bortezomib or vehicle. g) Heatmap representation of protein abundances in exosome-like vesicles from serum starved HUVECS cells treated with proteasome inhibitor bortezomib or vehicle. Bortezomib treatment strongly modulates the protein content of exosome-like vesicles. Perlecan is enriched in bortezomib-treated cells. h) LG3 spectral count in exosome-like vesicles from serum starved HUVECS treated with the proteasome inhibitor bortezomib or vehicle. i) Proteasome inhibition with Bortezomib results in the accumulation of ubiquitylated proteins in exosome-like vesicles. Upon bortezomib treatment 20 ubiquitylation sites on 19 proteins were detected, while in vehicle-treated control cells only 5 ubiquitinylation sites on 5 proteins were detected by mass spectrometry. mean \pm SEM, , t-test, ns=nonsignificant.

\section{Figure 5. Proteasome activity within apoptotic exosome-like vesicles regulates anti-LG3 autoantibody formation and allograft inflammation.}

a) Anti-LG3 IgG titers in sera from WT (grey bars) or allografted mice (black bars) after 3 weeks of i.v. injections with vehicle, or with exosome-like vesicles from serum starved murine ECs treated with the proteasome inhibitor bortezomib (Exo-like Bortezo) or vehicle (Exolike Vehicle). b) CD3, CD20 and C4d stained aortic allograft sections from mice injected with exosome-like vesicles from serum starved murine ECs treated with bortezomib (Exo-like Bortezo) or vehicle (Exo-like Vehicle). (magnification: 20X ) c) Mean number of CD3 positive cells (T cells) per high power field in allografts from mice injected with exosome-like vesicles from serum starved murine ECs treated with bortezomib (Exo-like Bortezo) or vehicle (Exo-like Vehicle), for 3 weeks post-transplantation. d) Mean number of CD20 positive cells (B cells) per high power field in allografts from mice injected with exosome-like vesicles from serum starved murine ECs treated with bortezomib (Exo-like Bortezo) or vehicle (Exo-like Vehicle), for 3 weeks posttransplantation. e) C4d deposition in allografts from mice injected with exosome-like vesicles from serum starved murine ECs treated with proteasome inhibitor bortezomib (Exo-like Bortezo) or vehicle (Exo-like Vehicle), for 3 weeks post-transplantation. $\mathrm{n} \geq 9$ for each condition. Data are pooled from 4 independent experiments. mean \pm SEM, if not indicated comparison with vehicle, t-test.

\section{Figure 6. Renal injury enhances circulating proteasome caspase-like activity, LG3 levels and anti-LG3 production}

a) Blood urea nitrogen (BUN) concentration in mice pre-surgery or at 2, 7 or 21 days post-renal ischemia/ reperfusion $(\mathrm{I} / \mathrm{R})$ injury. b) Serum creatinine concentration in mice pre-surgery or at 2, 7 or 21 days post-renal I/R injury. c) Quantification of proteasome caspase-like activity in exosomelike vesicles from serum pre-surgery or 2 days postrenal I/R injury. d) Serum anti-LG3 IgG titers pre-surgery or at 2, 714 and 21 days postrenal I/R injury e) Densitometry analyses of immunoblots for LG3 detection performed on exosome-like vesicles purified from serum of mice presurgery or 2 days post-renal I/R injury f) Representative Ponceau Red staining and immunoblot showing the detection of perlecan fragments, including LG3, in serum exosome-like vesicles pre surgery or 2 days post-renal I/R injury. g) Left panel: Electron micrographs of exosome-like vesicles isolated from serum 2 days post-renal ischemiareperfusion $(\mathrm{I} / \mathrm{R})$ injury. Right panels: Electron micrographs showing immunogold labeling for the alpha 3 proteasome subunit in exosome-like vesicles isolated from serum 2 days post-renal ischemia-reperfusion (I/R) injury. from $n=6$ for each condition, mean \pm SEM, comparison with pre-surgery. t-test.

\section{Figure 7. Vascular injury enhances circulating proteasome caspase-like activity, LG3 levels and anti-LG3 production}

a) Laser Doppler imaging of blood flow in the lower limbs of mice on the day of the surgery (day 0 ) or at 7 or 21 days post femoral arteriectomy (vascular injury) b) Quantification of proteasome caspase-like activity in serum exosomelike vesicles pre-surgery or 2 days post vascular injury c) Representative Ponceau Red staining and immunoblot showing the detection of perlecan fragments, including LG3, in serum exosome-like vesicles pre-surgery or 2 days post vascular injury. d) Densitometry analyses of immunoblots for LG3 detection performed on serum exosomelike vesicles pre surgery or 2 days post vascular injury. e) Serum anti-LG3 IgG titers pre-surgery or at 7, 14 and 21 days post vascular injury. $n=6$ for each condition, mean \pm SEM, comparison with pre-surgery, $t$-test. 